\title{
Diet quality as assessed by the Healthy Food Intake Index and relationship with serum lipoprotein particles and serum fatty acids in pregnant women at increased risk for gestational diabetes
}

\author{
Anita J. Valkama ${ }^{1,2 *}$, Jelena M. Meinilä ${ }^{1}$, Saila B. Koivusalo ${ }^{3}$, Jaana Lindström ${ }^{4}$, Kristiina Rönö ${ }^{3}$, \\ Beata Stach-Lempinen ${ }^{5}$ and Johan G. Eriksson ${ }^{1,2,4}$ \\ ${ }^{1}$ Department of General Practice and Primary Health Care, University of Helsinki and Helsinki University Hospital, \\ Tukbolmankatu 8 B, P.O. Box 20, O0014 Helsinki, Finland \\ ${ }^{2}$ Folkhälsan Research Center, University of Helsinki, Topeliuksenkatu 20, 00250 Helsinki, Finland \\ ${ }^{3}$ Department of Obstetrics and Gynecology, University of Helsinki and Helsinki University Hospital, Sofianlehdonkatu 5A, \\ P.O. Box 610, 00029 Helsinki, Finland \\ ${ }^{4}$ Department of Chronic Disease Prevention, National Institute for Health and Welfare, Mannerheimintie 166, P.O. Box 30, \\ 00271 Helsinki, Finland \\ ${ }^{5}$ Department of Obstetrics and Gynecology, South Karelia Central Hospital, Valto Käkelän Katu 3, 53130 Lappeenranta, \\ Finland
}

(Submitted 13 February 2018 - Final revision received 23 July 2018 - Accepted 1 August 2018)

\section{Abstract}

The importance of overall diet in modifying circulating lipoprotein particles and fatty acids during pregnancy is unclear. We examined the relationships of diet quality as assessed by the validated Healthy Food Intake Index (HFII) with serum HDL, LDL and VLDL particle concentrations and sizes and proportions of serum fatty acids in pregnant women at high risk for gestational diabetes mellitus (GDM). Overall, 161 women with a BMI of $\geq 30 \mathrm{~kg} / \mathrm{m}^{2}$ and/or a history of GDM were drawn from the Finnish Gestational Diabetes Prevention Study, which is a dietary and exercise intervention trial to prevent GDM. At baseline, the HFII score was inversely related to concentrations of HDL particles $(P=0.010)$ and MUFA $(P=0.010)$ and positively related to concentrations of $n-3(P<0 \cdot 001)$ and $n-6(P=0.003)$ PUFA. The significance for MUFA disappeared after adjustments. An increase in the HFII score from the first to second trimester of pregnancy correlated with reduced VLDL particle size $(r-0 \cdot 16,95 \%$ CI $-0 \cdot 31,-0 \cdot 01)$, decreased MUFA concentrations $(r-0 \cdot 17,95 \%$ CI $-0 \cdot 31,-0 \cdot 01)$ and elevated $n-6$ PUFA concentrations $(r 0 \cdot 16,95 \%$ CI $0 \cdot 01,0 \cdot 31)$. In the maximum-adjusted model, the results remained significant except for VLDL particle size. These findings suggest that higher diet quality as defined by the HFII is related to a more favourable serum fatty acid profile, whereas the relationship with serum lipoprotein profile is limited in pregnant women at increased GDM risk.

Key words: Diet quality: Lipoprotein particles: Serum fatty acids: Pregnancy: Gestational diabetes

Pregnancy is characterised by increased insulin resistance and several other metabolic alterations to support fetal growth ${ }^{(1)}$. Lipid metabolism in pregnancy is influenced by both anabolic and catabolic stages, which eventually lead to a more atherogenic lipid profile ${ }^{(2)}$. Obesity magnifies pregnancy-induced insulin resistance and metabolic changes, and increases the risk of pregnancy complications, including gestational diabetes mellitus $(\mathrm{GDM})^{(3,4)}$. In addition to obesity, also GDM is characterised by increased insulin resistance and has been related to a more unfavourable lipid profile ${ }^{(5)}$, as well as an increased risk of $\mathrm{CVD}^{(6)}$ in later life.
Evaluation of circulating lipoprotein particle profile is an alternative method to standard lipid testing in prediction of CVD and takes into account the heterogeneity in particle size and density of the lipoprotein subclasses ${ }^{(7)}$. In observational studies on nonpregnant populations, elevated concentrations and smaller size of LDL particles, elevated concentrations and larger size of VLDL particles, as well as lower concentrations and smaller size of HDL particles, have been related to increased insulin resistance and/or a higher risk of type 2 diabetes (T2D) and $\mathrm{CVD}^{(7-9)}$. Moreover, in pregnant populations, larger VLDL size has been associated with recurrent preterm birth and earlier gestational age at delivery ${ }^{(10,11)}$.

Abbreviations: GDM, gestational diabetes mellitus; HFII, Healthy Food Intake Index; NNR, Nordic Nutrition Recommendations; OGTT, oral glucose tolerance test; RADIEL, Finnish Gestational Diabetes Prevention Study; T2D, type 2 diabetes.

*Corresponding author: A. J. Valkama, email anita.valkama@helsinki.fi 
With regard to circulating fatty acids, several fatty acids have been proposed as novel biomarkers in cardiovascular risk prediction $^{(12,13)}$. Circulating $n-3$ PUFA have been associated with reduced CVD risk ${ }^{(14)}$. Moreover, in a recent meta-analysis including data from three population-based cohort studies, higher serum MUFA concentrations were associated with increased risk, whereas higher $n-6$ PUFA concentrations were associated with reduced risk of $\mathrm{CVD}^{(12)}$.

Healthy diet is one of the major factors contributing to cardiovascular health ${ }^{(15)}$. A few studies in non-pregnant populations suggest that healthy dietary patterns could modify lipoprotein profiles to less atherogenic by influencing lipoprotein particle concentrations and sizes. For instance, an adherence to a Mediterranean diet supplemented with nuts resulted in an increase in LDL particle size in a population at high CVD risk $^{(16)}$, whereas a 4-week lean-seafood intervention led to a reduced VLDL particle size in healthy individuals ${ }^{(17)}$. Furthermore, diet enriched with pistachio for 4 months resulted in reduced small LDL particle concentration compared with a nutfree $\operatorname{diet}^{(18)}$.

In addition to the wide use of circulating fatty acids as biomarkers of dietary fat intake, studies on food intakes have reported strong correlations between fish intake and circulating $n-3$ PUFA concentrations in non-pregnant populations ${ }^{(19,20)}$. Furthermore, findings from a large sub-study of the European Prospective Investigation into Cancer and Nutrition cohort revealed a strong correlation between olive oil consumption and circulating oleic acid ${ }^{(19)}$. However, data on whole diet approach and circulating fatty acids are limited and have mostly focused on the adherence to a Mediterranean dietary pattern $^{(21-23)}$. To our knowledge, no study has reported on the relationships of dietary patterns in Nordic countries with lipoprotein particle concentration and size and serum fatty acid concentrations in pregnancy affected by obesity and/or previous GDM.

In the present work, we investigated whether higher overall diet quality as evaluated by the adherence to Nordic Nutrition Recommendations (NNR) by using the Healthy Food Intake Index (HFII) is related to improvements in alternative lipid parameters in cardiovascular risk assessment. The aim was to explore whether higher diet quality is related to less atherogenic serum lipoprotein particle concentrations and sizes and fatty acid concentrations during early and middle pregnancy in Finnish pregnant women with increased risk of GDM.

\section{Methods}

\section{Study design and participants}

The present study was a sub-study of the randomised controlled Finnish Gestational Diabetes Prevention Study (RADIEL) trial, which was implemented in four maternity hospitals from 2008 to 2014. The main aim of the RADIEL trial was to investigate whether prevention of GDM is possible through lifestyle intervention initiated either before pregnancy or in early pregnancy ( $<20$ weeks of gestation) among women at increased risk for GDM owing to obesity and/or a history of GDM. The RADIEL trial was conducted according to the guidelines laid down in the
Declaration of Helsinki, and all procedures were approved by the Ethics Committees of Helsinki University Central Hospital and the South-Karelia Central Hospital. All participants consented to participate in the study.

In addition to standard visits at the maternity clinics, all women in the RADIEL trial visited a study nurse three times during pregnancy. In addition, women entering the RADIEL trial before pregnancy ( $n 33$ in the present study) participated in the study visits every 3 months before conception. The mean number of pre-pregnancy visits was $2 \cdot 1$ (range 1-4) in the present study. Women in the intervention group received individual dietary and exercise counselling from the study nurse during each study visit and, additionally, participated in a group counselling session with a dietitian after the first study visit. In addition, women with a pre-pregnancy BMI of $30 \mathrm{~kg} / \mathrm{m}^{2}$ were instructed to avoid weight gain during the first two trimesters of pregnancy. Dietary goals were to increase the consumption of vegetables, fruits and berries, as well as whole-grain and low-fat dairy products, and to lower the consumption of foods high in sugar. The aim of the exercise counselling was to achieve at least $150 \mathrm{~min} /$ week of moderate-intensity exercise. Women in the control group received similar counselling as provided in the standard visits at antenatal clinics. In our previous publication including women entering the RADIEL trial during pregnancy, the incidence of GDM, which was the main outcome of the RADIEL trial, was significantly lower in the intervention group (14\%) compared with the control group (22\%) after adjusting for age, pre-pregnancy BMI, a history of GDM and weeks of gestation $(P=0.044)^{(24)}$. The RADIEL trial has been registered at clinicaltrials.gov (clinical trial reg. no. NCT01698385). Further details of the design and methods in the RADIEL trial are available in a previous publication ${ }^{(25)}$.

The inclusion criteria were age $\geq 18$ years, a history of GDM and/or pre-pregnancy BMI of $\geq 30 \mathrm{~kg} / \mathrm{m}^{2}$. The exclusion criteria of the present study were similar to those reported in our previous publication ${ }^{(24)}$ : T2D diagnosed before pregnancy, diagnosis of GDM in the first trimester of pregnancy ( $<20$ weeks of gestation), physical disability, use of medication that influences glucose metabolism, multiple pregnancy, notable difficulty in co-operation (such as insufficient language skills), current substance abuse and severe psychiatric disorder. In addition, participants with no study visits during gestation (pre-pregnant women), no pregnancy within a year after recruitment (prepregnant women), and incomplete oral glucose tolerance test (OGTT), metabolic and/or dietary data in the first and/or second trimesters of pregnancy were excluded from this study. Diagnosis of GDM was based on at least one pathological plasma glucose value in a 2 -h 75 -g OGTT, and the thresholds were based on the values standardised by the American Diabetes Association (ADA): fasting plasma glucose, $\geq 5.3 \mathrm{mmol} / \mathrm{l}$; 1 -h glucose, $\geq 10.0 \mathrm{mmol} / \mathrm{l}$; and/or 2 -h glucose, $\geq 8.6 \mathrm{mmol} / \mathrm{l}^{(26)}$. T2D before pregnancy was diagnosed based on the following criteria: fasting plasma glucose $\geq 7.0 \mathrm{mmol} / 1$ and glycated $\mathrm{Hb}$ (HbA1c) $\geq 48 \mathrm{mmol} / 1$, or 2 -h plasma glucose $\geq 11.1 \mathrm{mmol} / 1$ in a 75-g OGTT. According to ADA criteria, one pathologic value is sufficient for T2D diagnosis ${ }^{(27)}$. However, in the absence of unequivocal hyperglycaemia, results should be confirmed by repeat testing. As only part of the T2D cases may be recognised 
based on either fasting plasma glucose or HbA1c measurement alone ${ }^{(28)}$, data from both measurements were required in order to exclude potential T2D in the present study.

None of the participants included in the present study used lipid-lowering medication or other medication influencing lipid metabolism.

In total, 787 women were recruited before or during pregnancy in the RADIEL trial. Overall, 720 pre-pregnant and pregnant women were randomised into control $(n$ 228) and intervention groups ( $n$ 492) in the RADIEL trial, of whom 161 were included in this study after exclusions: dropped out before pregnancy ( $n 11)$, no pregnancy within a year after recruitment ( $n$ 85), miscarriage or termination of pregnancy ( $n$ 17), multiple pregnancies ( $n$ 4), incomplete OGTT or HbA1c data in prepregnancy ( $n$ 29), incomplete OGTT data during pregnancy ( $n$ 68), pathologic OGTT in the first trimester of pregnancy ( $n$ 192) and incomplete metabolomics ( $n$ 131) or dietary index ( $n$ 22) data in the first and/or second trimester of pregnancy. The intervention and control groups, as well as women recruited before and during pregnancy, were combined to assess the relationships between the HFII and serum lipoprotein particles and serum fatty acids in pregnancy. Women who entered the RADIEL trial before pregnancy were followed up during pregnancy similarly to those who entered the RADIEL trial when pregnant. The data collection at the study visits was therefore performed in each trimester of pregnancy for all women in the RADIEL trial. In the present study, the participants were followed up from the first (6-17 weeks of gestation) to the second (20-27 weeks of gestation) trimester of pregnancy. The first trimester is considered as baseline henceforth.

\section{Baseline characteristics}

Baseline data of the present study refer to the data collected in the first trimester of pregnancy from all women. The data were obtained by means of questionnaires, except for anthropometric, laboratory and dietary data. The questionnaire-based amount of leisure-time physical activity was reported as weekly minutes of exercise that makes one at least slightly out of breath and sweaty. The participants were weighted and blood was drawn by a study nurse at each study visit. Weighing was performed with light clothing and no shoes. A study nurse measured blood pressure from the right arm by using a sphygmomanometer while the participant was sitting. Laboratory measurements were performed in the Helsinki University Hospital laboratory by using the following methods: enzymatic hexokinase assay (Gluco-quant, modular analyser; Roche Diagnostics) for fasting plasma glucose and electrochemiluminescence immunoassay (insulin, modular analyser) for fasting insulin. Energy intakes were estimated on the basis of a 3-d food record by two nutritionists using the food calculation software AivoDiet (Aivo Ltd), which in turn applies the Fineli Finnish Food Composition Database (National Institute for Health and Welfare, Nutrition Unit, Helsinki, Finland). The energy intake across the HFII groups was reported to demonstrate that energy intake was similar across the HFII groups, and that the HFII rather reflects diet quality instead of quantity.

\section{Serum lipid measurements}

Fasting blood samples were collected during the study visits in the first and second trimesters of pregnancy. Blood samples were frozen for up to $2 \mathrm{~h}$ in blood collection tubes and centrifuged at 2540 relative centrifugal force for $15 \mathrm{~min}$ at $4^{\circ} \mathrm{C}$. Serum samples were stored at $-80^{\circ} \mathrm{C}$ until analysis. Evaluation of serum lipoprotein particles and serum fatty acids was performed by using a highthroughput serum NMR spectroscopy metabolomics platform (Brainshake Ltd). NMR is a quick, cost-effective and reproducible methodology for metabolic profiling based on molecule-specific signals ${ }^{(29)}$. NMR is established to be suitable for quantification of lipoproteins ${ }^{(30,31)}$, and has been used in numerous epidemiological studies ${ }^{(32-35)}$. Measurements for lipoprotein particles included fasting concentrations (nmol/l and $\mu \mathrm{mol} / \mathrm{l}$ ) and size (nm) of VLDL, LDL and HDL particles. A less atherogenic lipoprotein particle profile was defined as lower concentrations and smaller size of VLDL particles, lower concentrations and larger size of LDL particles and higher concentrations and larger size of HDL particles. As small and medium LDL particles are more strongly associated with CVD risk compared with large LDL particles ${ }^{(36,37)}$, larger LDL particle size was considered preferential in the present study. Serum fatty acid profile was reported as the percentage of total fatty acids, and included total n-3 PUFA, total $n-6$ PUFA, MUFA and SFA. A more favourable serum fatty acid profile was defined as higher proportions of $n-3$ and $n-6$ PUFA, and lower proportions of MUFA and SFA. To further assess the relationship between diet quality and lipid metabolism in pregnancy, we also evaluated the serum concentrations of standard lipid parameters including TAG, total cholesterol, LDL-cholesterol and HDL-cholesterol. Methods used to evaluate the concentrations of standard lipid parameters were as follows: enzymatic assay (TAG GPO-PAP, modular analyser) for TAG, enzymatic assay (cholesterol CHOD-PAP, modular analyser) for total cholesterol, enzymatic assay (LDL-cholesterol plus 2nd generation, modular analyser) for LDL-cholesterol and enzymatic assay (HDL-cholesterol plus 3rd generation, modular analyser) for HDL-cholesterol. Changes in the lipid parameters were determined as subtracting the value in the first trimester from the value in the second trimester.

\section{Dietary measurements}

Diet quality was assessed by the adherence to the NNR by using the validated HFII based on a forty-eight-item FFQ used in the RADIEL trial. The FFQ was collected during the first- and second-trimester study visits. The HFII was created to assess the adherence to the $\mathrm{NNR}^{(38)}$ and the overall diet quality. Foods selected from the FFQ were further combined into eleven food groups: low-fat milk ( $\leq 1 \%$ fat), low-fat cheese ( $\leq 17 \%$ fat), vegetables and legumes, fruits and berries, whole grain (includes rye bread, porridge and brown rice and pasta), fish, snacks (includes candy, chocolate, pastries, chips and ice cream), fast food (includes hamburger and pizza), sugarsweetened beverages (includes sugar-sweetened juice and soft drinks), fat spread and cooking fat. Maximum score for each food group was either 1 or 2 based on the relevance in the overall diet quality. The eleven food groups were scored as follows: low-fat milk, 0-2; low-fat cheese, $0-1$; vegetables and 
legumes, $0-2$; fruits and berries, $0-1$; whole grain, $0-2$; fish, 0 or 2; snacks, $0-2$; fast food, $0-1$; sugar-sweetened beverages, $0-1$; fat spread, $0-2$; and cooking fat, $0-1$. The scoring for fat spread was as follows: margarine or low-fat margarine, 2 points; sterol margarine, 1 point; butter, butter-oil mix or no spreadable fat, 0 points. The scoring for cooking fat was as follows: vegetable oil (such as rapeseed and olive oil), margarine, liquid margarine or no cooking fat, 1 point; butter, butter-oil mix or baking margarine, 0 points. As a result, total HFII score ranged between 0 and 17 points, with higher total score indicating higher diet quality. Further details of the development, scoring and validation of the HFII are reported in a previous publication ${ }^{(39)}$ in which validation against 3-d food records showed that the HFII is valid for ranking pregnant participants according to adherence to the NNR. Change in the HFII was determined by subtracting the HFII score in the first trimester from the HFII score in the second trimester.

\section{Statistical analyses}

The participants were stratified into three groups according to the HFII score in the first trimester of pregnancy by using $z$ cutoff limits at $\pm 1 \mathrm{sD}$ from the mean. Similar categorisation has been used in our previous validation study ${ }^{(39)}$. Differences between the HFII groups at baseline were analysed by using one-way ANOVA and $\chi^{2}$ test. Linearity between HFII groups was evaluated by ANOVA and ANCOVA. Covariates for analyses were selected based on previous literature: gestational age at blood collection, BMI, age, years of education and physical activity. In addition, further adjustments for allocation group, study group and gestational weight gain were performed. The study group referred to women who entered the RADIEL trial either before pregnancy or during pregnancy. In all, four analytical models were performed: unadjusted model (model 1); a model adjusted for age, BMI, gestational age and years of education (model 2); a model adjusted for the covariates in model 2 and physical activity (model 3); and a model adjusted for the covariates in model 2, the allocation group and the study group (model 4). Gestational weight gain was included in model 4 as a covariate in the analyses for change in the HFII and change in lipid parameters. Residual change from the first to the second trimester of the HFII score and serum lipoprotein particles and serum fatty acids were calculated by regressing the secondtrimester values onto the first-trimester values. Residual change represents the amount of change that is independent of the baseline value. Correlations between residual changes in the HFII score and residual changes in the serum lipid parameters were performed with Pearson's correlation coefficients. Multivariate linear regression analyses were also performed to evaluate the association between residual changes in the HFII and residual changes in lipid parameters. Results of adjusting for physical activity were reported separately as the physical activity data at baseline were available only for 145 women. The normality of the variables was tested by using the Shapiro-Wilk $W$-test. The bootstrap method was used in case of unknown theoretical distribution of the test statistics or violation of the assumptions (e.g. non-normality). Effect size was calculated by using the method of Cohen ${ }^{(40)}$. Stata 14.1 (StataCorp LP) was used for the analysis. $P$ value $<0.05$ was considered significant.

\section{Results}

\section{Baseline characteristics}

At baseline, the mean HFII score was 9.9 (sD 3.0) points, and the mean gestational age was 13.1 (SD 1.6) weeks. Out of 161 participants included, 129 (80\%) were overweight or obese. The prevalence of a history of GDM $(P=0.015)$ and years of education $(P=0.045)$ showed positive linearity, whereas total fat intake showed negative linearity $(P=0.028)$ across the HFII groups (Table 1).

Overall, thirty-three (20\%) women developed GDM during the follow-up from the first to the second trimester of pregnancy. Mean baseline BMI was lower in women with GDM, 28.4 (sD 6.4$) \mathrm{kg} / \mathrm{m}^{2}$, compared with women without GDM, $31 \cdot 6$ (sD 5.2$) \mathrm{kg} / \mathrm{m}^{2}(P=0.004)$. The prevalence of a history of GDM was higher among women with GDM compared with women without GDM (70 v. 36\%, P<0.001). According to the allocation group, eighteen (25\%) women in the control group and fifteen $(17 \%)$ women in the intervention group developed GDM during the study follow-up $(P=0 \cdot 234)$. From those who entered the RADIEL trial before pregnancy, six (18\%) women developed GDM, and from those who entered the RADIEL trial during pregnancy twenty-seven (21\%) women developed GDM during the follow-up $(P=0 \cdot 712)$.

\section{Linearity between the Healthy Food Intake Index score and serum lipoprotein particles and serum fatty acids at baseline}

Baseline concentrations and sizes of serum lipoprotein particles and proportions of serum fatty acids in different HFII groups are presented in Table 2 . The HFII score was inversely related to HDL particle concentrations before and after adjustments (Table 2). The proportions of $n-3 \quad(P<0.001)$ and $n-6$ $(P=0.003)$ PUFA were positively related and that of MUFA $(P=0.010)$ was inversely related to the HFII score. After adjustments, the results for $n-6$ and $n-3$ PUFA remained significant, except for $n-3$ PUFA when adjusting by physical activity, whereas the results for MUFA were no longer significant (Table 2). Moreover, the HFII score showed an inverse relationship with proportions of SFA after adjustments, except when adjusted for the allocation and the study group (Table 2). No other relationships between the HFII score and lipoprotein particles or serum fatty acids were observed at baseline. Interaction analysis showed non-significant interactions between the HFII and GDM for lipid parameters, indicating that diet-lipid relationships were not influenced by GDM status.

\section{Correlations between changes in the HFIl score and changes in serum lipoprotein particles and serum fatty acids}

The mean follow-up time and change in the HFII score from the first to the second trimester were 10.3 (SD 1.9) weeks and 0.22 (sD 2.23) points, respectively. Correlations between changes in the HFII and changes in serum concentrations and sizes of lipoprotein particles are shown in Fig. 1. Changes in the HFII score were inversely correlated with changes in VLDL particle 
Table 1. Baseline characteristics according to the Healthy Food Intake Index (HFII) score in the first trimester of pregnancy (Mean values and standard deviations; numbers and percentages; medians and interquartile ranges (IQR))

\begin{tabular}{|c|c|c|c|c|c|c|c|c|}
\hline & \multirow[b]{3}{*}{$n$} & \multicolumn{6}{|c|}{ HFIl score } & \multirow[b]{3}{*}{$P^{\star}$} \\
\hline & & \multicolumn{2}{|c|}{$0-7(n$ 35) } & \multicolumn{2}{|c|}{$8-12(n 77)$} & \multicolumn{2}{|c|}{ 13-17 (n 49) } & \\
\hline & & Mean & SD & Mean & SD & Mean & SD & \\
\hline HFII score & 161 & $5 \cdot 86$ & 1.44 & $9 \cdot 57$ & $1 \cdot 11$ & $13 \cdot 37$ & $1 \cdot 20$ & $<0.001$ \\
\hline Age (years) & 161 & $30 \cdot 8$ & 4.5 & $32 \cdot 0$ & $4 \cdot 7$ & $32 \cdot 1$ & 4.4 & 0.181 \\
\hline BMI $\left(\mathrm{kg} / \mathrm{m}^{2}\right)$ & 161 & $31 \cdot 6$ & $4 \cdot 3$ & 31.4 & $5 \cdot 7$ & $29 \cdot 6$ & $6 \cdot 0$ & 0.074 \\
\hline Weight (kg) & 161 & $86 \cdot 5$ & $14 \cdot 7$ & $87 \cdot 1$ & $16 \cdot 6$ & 81.9 & $20 \cdot 1$ & $0 \cdot 225$ \\
\hline Gestational age (weeks) & 161 & $12 \cdot 5$ & $2 \cdot 0$ & $13 \cdot 4$ & 1.4 & $13 \cdot 2$ & 1.6 & 0.079 \\
\hline Years of education & 161 & $13 \cdot 7$ & 1.9 & $14 \cdot 4$ & $2 \cdot 2$ & $14 \cdot 6$ & $2 \cdot 1$ & 0.045 \\
\hline \multicolumn{9}{|l|}{ OGTT $(\mathrm{mmol} / \mathrm{l})$} \\
\hline Fasting & 161 & 4.90 & $0 \cdot 23$ & $4 \cdot 89$ & 0.24 & $4 \cdot 88$ & 0.21 & 0.580 \\
\hline $1-h$ & 161 & $6 \cdot 88$ & 1.54 & $7 \cdot 20$ & $1 \cdot 33$ & $6 \cdot 77$ & $1 \cdot 39$ & 0.740 \\
\hline 2-h & 161 & $5 \cdot 70$ & 1.01 & 6.02 & 1.07 & $5 \cdot 56$ & $1 \cdot 28$ & 0.576 \\
\hline Systolic blood pressure (mmHg) & 159 & 121 & 11 & 122 & 14 & 123 & 13 & 0.357 \\
\hline Diastolic blood pressure (mmHg) & 159 & 74.9 & $7 \cdot 7$ & $76 \cdot 5$ & $9 \cdot 2$ & $77 \cdot 8$ & $9 \cdot 9$ & $0 \cdot 131$ \\
\hline TAG $(\mathrm{mmol} / \mathrm{l})$ & 161 & $1 \cdot 30$ & 0.49 & $1 \cdot 30$ & 0.56 & $1 \cdot 17$ & 0.41 & 0.175 \\
\hline Fasting insulin (mU/l) & 160 & 8.56 & $4 \cdot 2$ & $7 \cdot 93$ & $4 \cdot 1$ & $7 \cdot 36$ & $5 \cdot 51$ & 0.253 \\
\hline Total energy intake (kJ/d) & 142 & 7887 & 1862 & 8134 & 1845 & 7933 & 1757 & 0.919 \\
\hline Total energy intake (kcal/d) & 142 & 1885 & 445 & 1944 & 441 & 1896 & 420 & 0.919 \\
\hline Total fat intake (E\%) & 142 & $35 \cdot 6$ & $5 \cdot 8$ & $33 \cdot 7$ & $5 \cdot 8$ & $32 \cdot 4$ & $5 \cdot 8$ & 0.028 \\
\hline Intervention group & 161 & & & & & & & 0.461 \\
\hline$n$ & & 19 & & 39 & & 30 & & \\
\hline$\%$ & & 54 & & 51 & & 61 & & \\
\hline Previous deliveries (yes/no) & 161 & & & & & & & $0 \cdot 108$ \\
\hline$n$ & & 21 & & 45 & & 37 & & \\
\hline$\%$ & & 60 & & 58 & & 76 & & \\
\hline History of gestational diabetes & 161 & & & & & & & 0.015 \\
\hline$n$ & & 11 & & 30 & & 28 & & \\
\hline$\%$ & & 31 & & 39 & & 57 & & \\
\hline Parents' history of diabetes & 159 & & & & & & & 0.546 \\
\hline$n$ & & 7 & & 13 & & 12 & & \\
\hline$\%$ & & 21 & & 17 & & 25 & & \\
\hline Parents' history of CVD & 159 & & & & & & & 0.525 \\
\hline$n$ & & 15 & & 41 & & 25 & & \\
\hline$\%$ & & 44 & & 53 & & 52 & & \\
\hline Parents' history of hypertension & 159 & & & & & & & 0.741 \\
\hline$n$ & & 14 & & 38 & & 22 & & \\
\hline$\%$ & & 41 & & 49 & & 46 & & \\
\hline Current smoker & 161 & & & & & & & 0.387 \\
\hline$n$ & & 3 & & 2 & & 2 & & \\
\hline$\%$ & & 9 & & 3 & & 4 & & \\
\hline Physical activity (min/week) & 145 & & & & & & & 0.332 \\
\hline Median & & 60 & & 60 & & 90 & & \\
\hline IQR & & $25-135$ & & $30-120$ & & $45-145$ & & \\
\hline
\end{tabular}

OGTT, oral glucose tolerance test; E\%, percentage from total energy intake.

* Linearity across the HFII groups. ANOVA for continuous variables and Cochran-Armitage test for trend for categorical variables.

size $(r-0 \cdot 16,95 \% \mathrm{CI}-0 \cdot 31,-0 \cdot 01)$. In adjusted models, this correlation remained similar (model 2: $r-0 \cdot 15$, 95\% CI $-0 \cdot 29$, -0.01; model 3: $r-0 \cdot 17,95 \% \mathrm{CI}-0 \cdot 32,-0 \cdot 01)$, except in model 4 in which the correlation was slightly attenuated $(r-0 \cdot 15,95 \%$ CI $-0.30,0 \cdot 00)$. No other correlations were observed between changes in the HFII score and changes in lipoprotein particle concentrations and sizes. Correlations between changes in the HFII score and changes in proportions of serum fatty acids are shown in Fig. 2. Changes in the proportions of $n$ - 6 PUFA were positively correlated $(r 0 \cdot 16,95 \%$ CI $0 \cdot 01,0 \cdot 31)$ and those of MUFA were inversely correlated $(r-0 \cdot 17,95 \%$ CI $-0 \cdot 31,-0 \cdot 01)$ with changes in the HFII score. The results remained significant for both n-6 PUFA and MUFA after adjustments (data not shown). The effects sizes were rather small for all significant effects. Similarly, in multivariate linear regression models, the
HFII change was linearly associated with $n$-6 PUFA change and inversely associated with MUFA change (online Supplementary Table S1). The association between change in the HFII and change in VLDL particle size was only slightly attenuated after further adjustments $(P=0.05)$ (model 4 , online Supplementary Table S1). Moreover, change in standard lipid parameters showed no association with change in the HFII before and after adjustments (online Supplementary Tables S2 and S3).

\section{Discussion}

The present study explored whether diet quality as assessed by the adherence to the NNR based on the food-based HFII is related to serum lipoprotein particle concentrations and sizes 
Table 2. Baseline serum lipoprotein particle and serum fatty acid profiles across the Healthy Food Intake Index (HFII) groups in the first trimester of pregnancy $(n 161)$

(Mean values and standard deviations)

\begin{tabular}{|c|c|c|c|c|c|c|c|c|c|c|}
\hline & \multicolumn{6}{|c|}{ HFII score } & \multirow[b]{3}{*}{$P_{\text {for trend }}{ }^{*}$} & \multirow[b]{3}{*}{$P_{\text {for trend }} \dagger$} & \multirow[b]{3}{*}{$P_{\text {for trend }} \ddagger$} & \multirow[b]{3}{*}{$P_{\text {for trend }} \S$} \\
\hline & \multicolumn{2}{|c|}{$0-7(n 35)$} & \multicolumn{2}{|c|}{$8-12(n 77)$} & \multicolumn{2}{|c|}{$13-17(n 49)$} & & & & \\
\hline & Mean & SD & Mean & SD & Mean & SD & & & & \\
\hline \multicolumn{11}{|c|}{ Lipoprotein particle profile } \\
\hline \multicolumn{11}{|c|}{ Concentration } \\
\hline VLDL $(\mathrm{nmol} / \mathrm{l})$ & 79.9 & 24.5 & $80 \cdot 2$ & $26 \cdot 5$ & $77 \cdot 0$ & 24.4 & 0.590 & 0.627 & 0.947 & 0.705 \\
\hline LDL (nmol/l) & 546 & 131 & 538 & 131 & 563 & 152 & 0.585 & 0.977 & 0.878 & 0.968 \\
\hline $\mathrm{HDL}(\mu \mathrm{mol} / \mathrm{l})$ & $10 \cdot 1$ & 1.1 & 9.72 & 0.97 & 9.49 & 0.97 & 0.010 & $<0.001$ & 0.002 & 0.002 \\
\hline \multicolumn{11}{|l|}{ Size $(\mathrm{nm})$} \\
\hline VLDL & $36 \cdot 2$ & $1 \cdot 1$ & $36 \cdot 1$ & 1.2 & 35.9 & 1.0 & 0.247 & 0.745 & 0.892 & 0.805 \\
\hline LDL & $23 \cdot 6$ & 0.1 & $23 \cdot 6$ & 0.1 & $23 \cdot 6$ & 0.1 & 0.382 & 0.499 & 0.293 & 0.626 \\
\hline HDL & $10 \cdot 2$ & 0.2 & $10 \cdot 2$ & 0.2 & $10 \cdot 2$ & 0.2 & 0.986 & 0.329 & 0.272 & 0.330 \\
\hline \multicolumn{11}{|c|}{$\begin{array}{l}\text { Fatty acid profile (percentage } \\
\text { of total fatty acids) }\end{array}$} \\
\hline$n-3$ fatty acids & 4.22 & 0.58 & 4.51 & 0.66 & 4.74 & 0.83 & $<0.001$ & 0.013 & 0.056 & 0.020 \\
\hline$n-6$ fatty acids & $32 \cdot 1$ & 2.4 & 32.9 & 3.9 & 33.8 & 2.9 & 0.003 & 0.005 & 0.017 & 0.010 \\
\hline MUFA & $28 \cdot 3$ & $2 \cdot 3$ & $27 \cdot 0$ & $5 \cdot 3$ & $26 \cdot 6$ & $3 \cdot 8$ & 0.010 & 0.059 & $0 \cdot 177$ & 0.077 \\
\hline SFA & 35.5 & $1 \cdot 1$ & 35.5 & $2 \cdot 2$ & 34.9 & 1.6 & 0.088 & 0.039 & 0.001 & 0.062 \\
\hline
\end{tabular}

* Model 1: unadjusted. Linearity across the HFII groups was tested by using ANOVA and ANCOVA.

$\dagger$ Model 2: adjusted for age, BMI, years of education and gestational age. Linearity across the HFII groups was tested by using ANOVA and ANCOVA.

¥ Model 3: adjusted for age, BMI, years of education, gestational age and physical activity $(n 145)$. Linearity across the HFII groups was tested by using ANOVA and ANCOVA.

$\S$ Model 4: adjusted for age, BMl, years of education, gestational age, the allocation group and the study group (time when the participant entered the Finnish Gestational Diabetes Prevention Study trial; before or during pregnancy). Linearity across the HFII groups was tested by using ANOVA and ANCOVA.

and with serum fatty acid concentrations in pregnant women at high risk for GDM. Higher HFII score was related to lower concentration of HDL particles, greater proportions of serum $n$ 3 and $n-6$ PUFA, as well as to lower proportions of serum SFA at baseline. In addition, an increase in the HFII score was related to a decrease in VLDL particle size and proportions of serum MUFA, as well as with increased proportions of serum $n-6$ PUFA between the first and second trimester of pregnancy. In addition, the results for $n-3$ PUFA change almost reached a level of statistical significance. To our knowledge, this is the first study to report on the relationships of an overall diet quality based on the adherence to NNR with serum lipoprotein particle concentrations and sizes, as well as serum fatty acid concentrations in Nordic pregnant women affected by obesity and/ or prior GDM.

The inverse HDL particle concentration-diet relationship at baseline may partly result from lower total fat content in the $\operatorname{diet}^{(41)}$, which was also supported by the present findings of an inverse relationship of total fat intake and the HFII score at baseline. Studies on diet and circulating lipids have usually reported on the cholesterol concentration of lipoprotein particles instead of total lipoprotein particle concentrations. Some of these studies have shown similar results for HDL-cholesterol as the present findings for HDL particle concentration. In a metaanalysis including three cross-sectional Finnish studies, an inverse association was observed for HDL-cholesterol concentration and adherence to a healthy Nordic diet ${ }^{(42)}$. Furthermore, findings from observational data on the relationship between higher adherence to a healthy dietary pattern and lower HDL-cholesterol concentrations are supported by those from dietary intervention studies. For instance, after a 6-week healthy Nordic diet, plasma HDL-cholesterol was reduced by
$5 \%$ compared with the control diet in hypercholesterolaemic Swedish participants ${ }^{(43)}$. In addition, in a clinical trial in healthy men and women, the 3-week Dietary Approaches to Stop Hypertension (DASH) diet resulted in reduced concentrations of plasma HDL-cholesterol ${ }^{(44)}$. However, no effect was observed on plasma HDL particle concentrations after the DASH diet intervention. With regard to change in lipoprotein parameters, improvement in the HFII score was significantly yet weakly correlated with reduced VLDL particle size, and the correlation was only slightly attenuated in the maximumadjusted model. Larger VLDL particles carry more TAG than smaller VLDL particles and are associated with increased insulin resistance and a higher T2D risk ${ }^{(8,9)}$. Some studies suggest that higher fish intake may play a role in modification of VLDL particle size. A 4-week lean-seafood intervention compared with non-seafood intervention resulted in reduced VLDL particle size in non-pregnant, healthy Norwegian population ${ }^{(17)}$. In addition, higher fish intake and a higher serum DHA to total fatty acid ratio (a biomarker of fish intake) were linked to reduced VLDL particle size, whereas higher intake of junk food, such as French fries, salty snacks and sweets, was related to increased VLDL particle size in a cross-sectional study of young adult Finnish twins ${ }^{(45)}$. Similar to the above-mentioned findings for fish intake, higher $n$-3 PUFA intake was associated with lower concentrations of large VLDL particles and smaller VLDL particle mean size among Alaska Eskimos at increased risk for $\mathrm{CVD}^{(46)}$. Potential mechanisms underlying the inverse association observed between diet and VLDL particle size go beyond the scope of the present study. However, in the literature focusing on $n$-3 PUFA intakes, it has been proposed that $n-3$ PUFA may modify VLDL particle size by enhancing the expression of lipoprotein lipase resulting in increased removal 

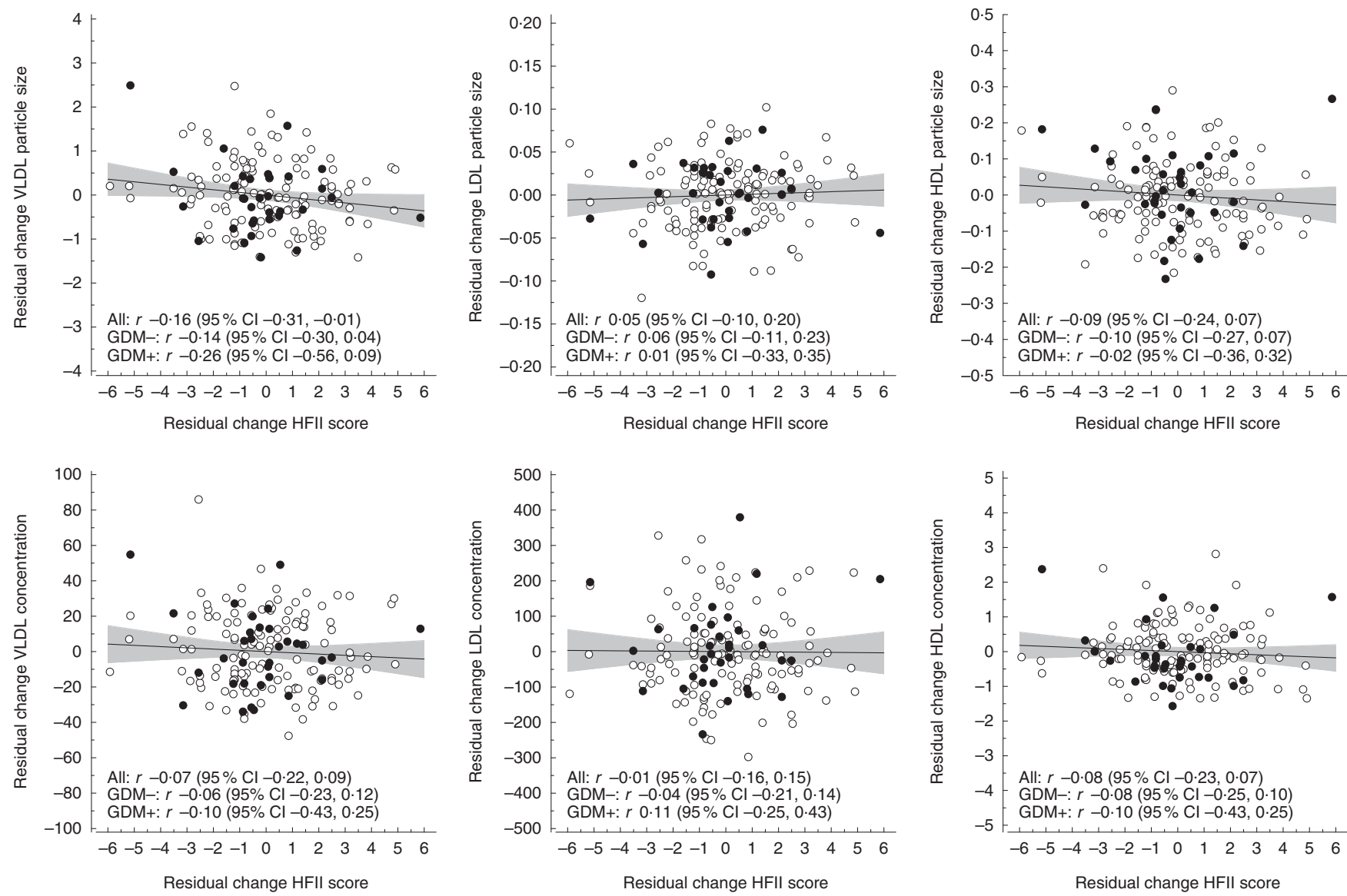

Fig. 1. Correlations between residual changes in the Healthy Food Intake Index (HFII) score and residual changes in serum lipoprotein particle concentration and size. The lines represent estimated linear regression with $95 \% \mathrm{Cl}$. GDM, gestational diabetes mellitus.

of TAG from the VLDL particles and further conversion to smaller VLDL particles and eventually to LDL particles ${ }^{(46,47)}$. On the other hand, in the present study, improvements in the HFII showed no association with change in LDL concentrations.

The rather weak relationships observed between lipoprotein particles and diet suggest that the role of diet is rather small and other factors than diet probably have more influence on maternal lipoprotein particle concentrations and sizes. For instance, pregnancy itself affects lipoproteins leading to a more atherogenic lipoprotein profile, such as decreased size of LDL particles and elevated VLDL particle concentrations ${ }^{(48,49)}$. Further, certain factors may contribute to the lipid response to dietary intake. Most of the women in the present study were overweight or obese (80\%). Dietary modifications such as adherence to low-SFA and low-cholesterol diets to improve serum lipid profile seem to be less effective in obese individuals compared with lean individuals, indicating that higher adiposity may attenuate the lipid response to dietary intake ${ }^{(50)}$. One mechanism for this might be obesity-related low-grade chronic inflammation, which results in increased insulin resistance ${ }^{(50)}$. Furthermore, the possible negative influence of insulin resistance on lipid response may be exaggerated during pregnancy. Another factor influencing circulating lipid response to diet is genetic polymorphism. For instance, the replacement of diet rich in carbohydrate with a diet rich in MUFA resulted in opposite effects on LDL particle size depending on the genotype of apoE in healthy young adults ${ }^{(51)}$. Similarly, higher saturated fat intake has been associated with either smaller or larger LDL particle size depending on the apoE genotype in Costa Rican men and women ${ }^{(52)}$. apoE is an important structural component in TAG-rich lipoprotein particles such as VLDL particles, as well as in HDL particles. Other frequently studied genetic factors influencing lipid response to diet are genetic polymorphisms in apoA4, apoB and lipoprotein lipase ${ }^{(53)}$. In addition, other factors contributing to lipid metabolism, but not included in the present study, may have influenced the findings.

The overall findings on serum fatty acids indicate that higher quality of diet as measured by the adherence to the NNR by using the HFII is related to a more favourable serum fatty acid profile in pregnant women at high risk for GDM. The significant results for $n-6$ PUFA change and non-significant results for $n-3$ PUFA change may in part arise from too small changes $n-3$ PUFA to show significant relationships, competition between $n-6$ and $n-3$ PUFA in physiologic processes, and the abundance of $n-6$ PUFA compared with $n-3$ PUFA in the western diet ${ }^{(54,55)}$. Furthermore, in addition to dietary intake, the proportions of $n-3$ and $n-6$ PUFA are also influenced by the enzyme activity of desaturases and elongases ${ }^{(56)}$.

Lower proportions of MUFA were considered favourable in the present study, as elevated circulating MUFA concentrations 

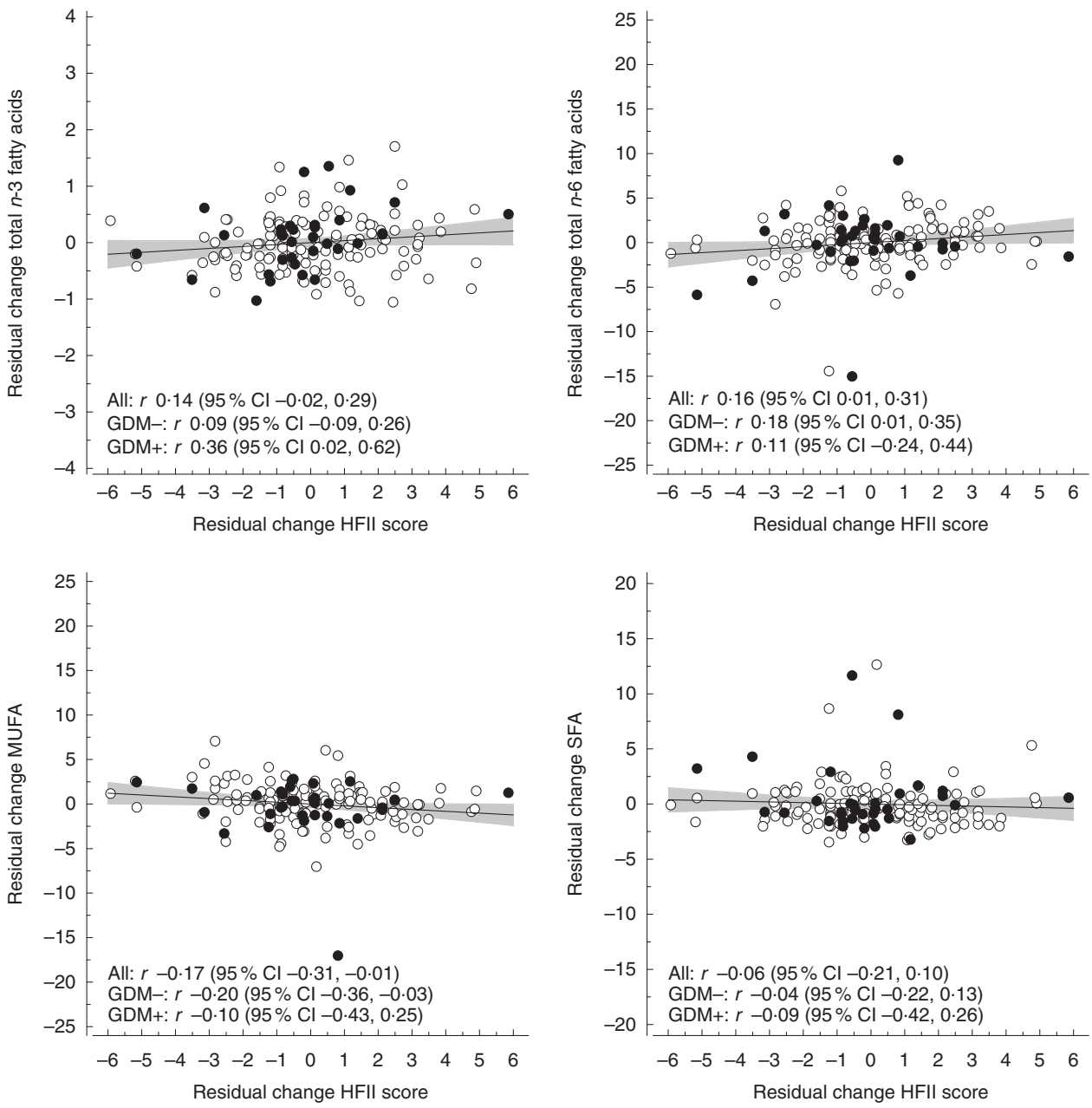

Fig. 2. Correlations between residual changes in the Healthy Food Intake Index (HFII) score and residual changes in proportions (percentage of total fatty acids) of serum fatty acids. The lines represent estimated linear regression with $95 \% \mathrm{Cl}$. GDM, gestational diabetes mellitus.

have been associated with a higher risk of cardiovascular event $^{(12)}$ and $\mathrm{T}_{2} \mathrm{D}^{(33)}$ in non-pregnant populations. On the other hand, a recent meta-analysis including prospective observational studies reported non-significant findings of the relationship between circulating MUFA and cardiovascular health ${ }^{(57)}$. With regard to dietary MUFA, data from the Nurses' Health Study revealed an inverse relationship between MUFA intake and CVD mortality ${ }^{(58)}$. Furthermore, in the Prevención con Dieta Mediterránea trial, the adherence to a Mediterranean diet enriched with either extra virgin olive oil or nuts, both of which are high in MUFA, showed beneficial effects on cardiovascular health in a population at CVD risk ${ }^{(59)}$. Further, in a pooled analysis of eleven cohort studies, substituting dietary MUFA for SFA was not associated with risk for $\mathrm{CHD}^{(60)}$. Overall, the evidence on circulating and dietary MUFA in relation to cardiovascular health is still inconsistent, which may partly arise from differences in the study design and populations, the endogenous MUFA synthesis, as well as different dietary sources of MUFA, namely animal- and plant-based sources. The expected inverse relationship between the HFII change and serum MUFA change is probably a consequence of that circulating MUFA derive from desaturation of dietary SFA ${ }^{(33)}$.
Similarly, the endogenous synthesis of SFA may partly explain the non-significant findings regarding the SFA change.

The HFII has been previously validated against 3-d food records ${ }^{(39)}$, which showed similar associations on dietary fatty acid intake as the corresponding serum fatty acids in the present study. The only exception was the positive association between MUFA intake (reported as percentage of total fatty acids) and the HFII observed in the validation study. The present study including serum concentrations of dietary fatty acids provides further validation for the HFII as a tool for dietary intake assessment. Traditional dietary measurement techniques are based on recording or recalling of the food intake, and thus these methods are susceptible to errors such as intentional under-reporting and dietary alterations during the recording days ${ }^{(56)}$. Therefore, the use of different biomarkers as a measure of dietary intake and nutrient status and as a predictor of disease risk provides a more accurate and objective approach to diet-disease associations ${ }^{(61)}$.

Strengths of the present study were detailed serum lipoprotein particle and serum fatty acid measurements in two different time points and the whole diet approach. Exploring the whole diet may improve understanding of the dietary influence in 
cardiovascular health, and the findings are transferable to public health recommendations and usable in clinical practice.

The present study has some limitations. The associations observed between the HFII and serum lipids were rather weak and must be considered with caution. In addition, the sample size may not have been sufficient to meet the requirements of statistical power. With regard to circulating MUFA, NMR measures the concentrations of both, palmitoleic acid (16:1) and oleic acid (18:1), and is unable to differentiate these subclasses. From dietary point of view, dietary palmitoleic and oleic acids may have opposite relationship with cardiovascular health ${ }^{(62)}$. However, owing to the endogenous synthesis ${ }^{(12)}$, circulating MUFA concentration is an inaccurate biomarker of dietary MUFA intake. In addition, the self-reported amount of weekly physical activity may have caused measurement bias. Furthermore, the dietary index used may not reflect all the dietary components and intakes that may influence circulating lipoprotein particle and fatty acid metabolism. For instance, the HFII was lacking a category of red and processed meat as different types of meat were not specified in the original version of the FFQ. Higher intake of red and processed meat has been associated with increased risk of T2D, CVD and GDM ${ }^{(63-66)}$. Thus, inclusion of this food group could have improved the HFII as a tool for assessing dietary intake and diet-circulating lipid relationships. Furthermore, the reported SD for change in the HFII score was rather high, $2 \cdot 23$, indicating wide variation from the mean change among the participants. These results can only be generalised to Caucasian pregnant women affected by obesity and/or a history of GDM. In addition, the results are based on Nordic pregnant population and may be different in other pregnant populations with different dietary culture and genetics.

In conclusion, higher diet quality as assessed by the adherence to NNR by using the HFII was related to a more favourable serum fatty acid profile, whereas the relationship with serum lipoproteins was limited in pregnant women at increased GDM risk. Overall, the findings suggest that the role of diet is rather small and other factors probably have greater importance in lipoprotein particle profiles during pregnancy. High-quality studies in pregnant women are needed to better understand the possibilities of dietary modification in improving maternal lipoprotein and fatty acid profiles and, by that means, in reducing the risk of pregnancy complications and future disease in the mother and the offspring. Moreover, studies on the factors influencing maternal circulating lipid response to dietary intake are required.

\section{Acknowledgements}

The authors thank all the participants for participating in the study, as well as the study personnel for contributing to the implementation of the study.

This study was a part of the RADIEL trial. The RADIEL trial was supported by Jalmari and Rauha Ahokas Foundation (J. G. E.), The Finnish Diabetes Research Foundation (J. G. E.), State Provincial Office of Southern Finland (J. G. E.), special state subsidy for health science research of Helsinki University Central Hospital (J. G. E.), Finnish Foundation for Cardiovascular Disease (J. G. E.),
Samfundet Folkhälsan (J. G. E.), the Social Insurance Institution of Finland and the Academy of Finland (J. G. E.) (grant no. 129369, $129907,135072,129255$ and 126775). The study resulting in these results has received funding from the European Commission within the 7th Framework Programme (DORIAN, grant agreement no. 278603) and EU H2020-PHC-2014-DynaHealth (grant no. 633595). The funders had no role in the design, analysis or writing of this article.

A. J. V., J. M. M., S. B. K., J. L., K. R., B. S.-L. and J. G. E. contributed to the design and implementation of the study; A. J. V. performed the statistical analyses, interpreted the results and wrote the first version of the manuscript; all authors read and approved the final version of the manuscript.

The authors declare that there are no conflicts of interest.

\section{Supplementary material}

For supplementary material/s referred to in this article, please visit https://doi.org/10.1017/S0007114518002404

\section{References}

1. Lain KY \& Catalano PM (2007) Metabolic changes in pregnancy. Clin Obstet Gynecol 50, 938-948.

2. Herrera E (2000) Metabolic adaptations in pregnancy and their implications for the availability of substrates to the fetus. EurJ Clin Nutr 54, Suppl. 1, S47-S51.

3. American Dietetic Association, American Society of Nutrition, Siega-Riz AM, et al. (2009) Position of the American Dietetic Association and American Society for Nutrition: obesity, reproduction, and pregnancy outcomes. J Am Diet Assoc 109, 918-927.

4. Catalano PM (2007) Management of obesity in pregnancy. Obstet Gynecol 109, 419-433.

5. Meyers-Seifer CH \& Vohr BR (1996) Lipid levels in former gestational diabetic mothers. Diabetes Care 19, 1351-1356.

6. Shah BR, Retnakaran R \& Booth GL (2008) Increased risk of cardiovascular disease in young women following gestational diabetes mellitus. Diabetes Care 31, 1668-1669.

7. Mora S, Otvos JD, Rifai N, et al. (2009) Lipoprotein particle profiles by nuclear magnetic resonance compared with standard lipids and apolipoproteins in predicting incident cardiovascular disease in women. Circulation 119, 931-939.

8. Festa A, Williams K, Hanley AJ, et al. (2005) Nuclear magnetic resonance lipoprotein abnormalities in prediabetic subjects in the Insulin Resistance Atherosclerosis Study. Circulation 111, 3465-3472.

9. Garvey WT, Kwon S, Zheng D, et al. (2003) Effects of insulin resistance and type 2 diabetes on lipoprotein subclass particle size and concentration determined by nuclear magnetic resonance. Diabetes 52, 453-462.

10. Thorp JM Jr, Rice MM, Harper M, et al. (2013) Advanced lipoprotein measures and recurrent preterm birth. Am J Obstet Gynecol 209, 342.e1-342.e7.

11. Grace MR, Vladutiu CJ, Nethery RC, et al. (2018) Lipoprotein particle concentration measured by nuclear magnetic resonance spectroscopy is associated with gestational age at delivery: a prospective cohort study. BJOG $\mathbf{1 2 5}$, 895-903.

12. Wurtz P, Havulinna AS, Soininen P, et al. (2015) Metabolite profiling and cardiovascular event risk: a prospective study of 3 population-based cohorts. Circulation 131, 774-785. 
13. Jackson KH \& Harris WS (2018) Blood fatty acid profiles: new biomarkers for cardiometabolic disease risk. Curr Atheroscler Rep 20, 22.

14. Matthan NR, Ooi EM, Van Horn L, et al. (2014) Plasma phospholipid fatty acid biomarkers of dietary fat quality and endogenous metabolism predict coronary heart disease risk: a nested case-control study within the Women's Health Initiative observational study. J Am Heart Assoc 3, e000764.

15. Lloyd-Jones DM, Hong Y, Labarthe D, et al. (2010) Defining and setting national goals for cardiovascular health promotion and disease reduction: the American Heart Association's strategic impact goal through 2020 and beyond. Circulation 121, 586-613.

16. Damasceno NR, Sala-Vila A, Cofan M, et al. (2013) Mediterranean diet supplemented with nuts reduces waist circumference and shifts lipoprotein subfractions to a less atherogenic pattern in subjects at high cardiovascular risk. Atherosclerosis 230, 347-353.

17. Aadland EK, Lavigne C, Graff IE, et al. (2015) Lean-seafood intake reduces cardiovascular lipid risk factors in healthy subjects: results from a randomized controlled trial with a crossover design. Am J Clin Nutr 102, 582-592.

18. Hernandez-Alonso P, Salas-Salvado J, Baldrich-Mora M, et al. (2015) Effect of pistachio consumption on plasma lipoprotein subclasses in pre-diabetic subjects. Nutr Metab Cardiovasc Dis 25, 396-402.

19. Saadatian-Elahi M, Slimani N, Chajes V, et al. (2009) Plasma phospholipid fatty acid profiles and their association with food intakes: results from a cross-sectional study within the European Prospective Investigation into Cancer and Nutrition. Am J Clin Nutr 89, 331-346.

20. Astorg P, Bertrais S, Laporte F, et al. (2008) Plasma $n-6$ and $n-3$ polyunsaturated fatty acids as biomarkers of their dietary intakes: a cross-sectional study within a cohort of middle-aged French men and women. Eur J Clin Nutr 62, 1155-1161.

21. Feart C, Torres MJ, Samieri C, et al. (2011) Adherence to a Mediterranean diet and plasma fatty acids: data from the Bordeaux sample of the Three-City study. Br J Nutr 106, 149-158.

22. Djuric Z, Ren J, Blythe J, et al. (2009) A Mediterranean dietary intervention in healthy American women changes plasma carotenoids and fatty acids in distinct clusters. Nutr Res 29, $156-163$

23. Itsiopoulos C, Brazionis L, Kaimakamis M, et al. (2011) Can the Mediterranean diet lower HbA1c in type 2 diabetes? Results from a randomized cross-over study. Nutr Metab Cardiovasc Dis 21, 740-747.

24. Koivusalo SB, Rono K, Klemetti MM, et al. (2016) Gestational diabetes mellitus can be prevented by lifestyle intervention: the Finnish Gestational Diabetes Prevention Study (RADIEL): a randomized controlled trial. Diabetes Care 39, 24-30.

25. Rono K, Stach-Lempinen B, Klemetti MM, et al. (2014) Prevention of gestational diabetes through lifestyle intervention: study design and methods of a Finnish randomized controlled multicenter trial (RADIEL). BMC Pregnancy Childbirth 14, 70.

26. American Diabetes Association (2008) Standards of medical care in diabetes-2008. Diabetes Care 31, Suppl. 1, S12-S54.

27. American Diabetes Association (2014) Diagnosis and classification of diabetes mellitus. Diabetes Care 37, Suppl. 1, S81-S90.

28. Wang W, Lee ET, Howard BV, et al. (2011) Fasting plasma glucose and hemoglobin A1c in identifying and predicting diabetes: the strong heart study. Diabetes Care 34, 363-368.

29. Soininen P, Kangas AJ, Wurtz P, et al. (2015) Quantitative serum nuclear magnetic resonance metabolomics in cardiovascular epidemiology and genetics. Circ Cardiovasc Genet $\mathbf{8}$, 192-206.
30. Ala-Korpela M, Korhonen A, Keisala J, et al. (1994) 1H NMRbased absolute quantitation of human lipoproteins and their lipid contents directly from plasma. J Lipid Res 35, 2292-2304.

31. Mihaleva VV, van Schalkwijk DB, de Graaf AA, et al. (2014) A systematic approach to obtain validated partial least square models for predicting lipoprotein subclasses from serum NMR spectra. Anal Chem 86, 543-550

32. Inouye M, Kettunen J, Soininen P, et al. (2010) Metabonomic, transcriptomic, and genomic variation of a population cohort. Mol Syst Biol 6, 441.

33. Mahendran Y, Cederberg H, Vangipurapu J, et al. (2013) Glycerol and fatty acids in serum predict the development of hyperglycemia and type 2 diabetes in Finnish men. Diabetes Care 36, 3732-3738.

34. Fischer K, Kettunen J, Wurtz P, et al. (2014) Biomarker profiling by nuclear magnetic resonance spectroscopy for the prediction of all-cause mortality: an observational study of 17,345 persons. PLoS Med 11, e1001606.

35. Wurtz P, Wang Q, Kangas AJ, et al. (2014) Metabolic signatures of adiposity in young adults: Mendelian randomization analysis and effects of weight change. PLoS Med 11, e1001765.

36. Williams PT, Zhao XQ, Marcovina SM, et al. (2014) Comparison of four methods of analysis of lipoprotein particle subfractions for their association with angiographic progression of coronary artery disease. Atherosclerosis 233, 713-720.

37. St-Pierre AC, Cantin B, Dagenais GR, et al. (2005) Low-density lipoprotein subfractions and the long-term risk of ischemic heart disease in men: 13-year follow-up data from the Quebec Cardiovascular Study. Arterioscler Thromb Vasc Biol 25, $553-559$

38. Nordic Council of Ministers (2014) Nordic Nutrition Recommendations 2012 - Integrating Nutrition and Physical Activity, 5th ed. Copenhagen: Nordic Council of Ministers.

39. Meinila J, Valkama A, Koivusalo SB, et al. (2016) Healthy Food Intake Index (HFII) - validity and reproducibility in a gestationaldiabetes-risk population. BMC Public Health 16, 680.

40. Cohen J (1988) Statistical Power Analysis for the Behavioral Sciences, 2nd ed. Hillsdale, NJ: Lawrence Erlbaum Associates.

41. Sacks FM \& Katan M (2002) Randomized clinical trials on the effects of dietary fat and carbohydrate on plasma lipoproteins and cardiovascular disease. Am J Med 113, Suppl. 9B, 13S-24S.

42. Kanerva N, Kaartinen NE, Rissanen H, et al. (2014) Associations of the Baltic Sea diet with cardiometabolic risk factors-a meta-analysis of three Finnish studies. Br J Nutr 112, 616-626.

43. Adamsson V, Reumark A, Fredriksson IB, et al. (2011) Effects of a healthy Nordic diet on cardiovascular risk factors in hypercholesterolaemic subjects: a randomized controlled trial (NORDIET). J Intern Med 269, 150-159.

44. Chiu S, Bergeron N, Williams PT, et al. (2016) Comparison of the DASH (Dietary Approaches to Stop Hypertension) diet and a higher-fat DASH diet on blood pressure and lipids and lipoproteins: a randomized controlled trial. Am J Clin Nutr 103, 341-347.

45. Bogl LH, Pietilainen KH, Rissanen A, et al. (2013) Association between habitual dietary intake and lipoprotein subclass profile in healthy young adults. Nutr Metab Cardiovasc Dis 23, 1071-1078.

46. Annuzzi G, Rivellese AA, Wang H, et al. (2012) Lipoprotein subfractions and dietary intake of $n-3$ fatty acid: the Genetics of Coronary Artery Disease in Alaska Natives study. Am J Clin Nutr 95, 1315-1322.

47. Dias CB, Amigo N, Wood LG, et al. (2017) Effect of diets rich in either saturated fat or $n-6$ polyunsaturated fatty acids and 
supplemented with long-chain $n-3$ polyunsaturated fatty acids on plasma lipoprotein profiles. Eur J Clin Nutr $\mathbf{7 1}$, 1297-1302.

48. Hubel CA, Shakir Y, Gallaher MJ, et al. (1998) Low-density lipoprotein particle size decreases during normal pregnancy in association with triglyceride increases. J Soc Gynecol Investig 5, 244-250.

49. Sattar N, Greer IA, Louden J, et al. (1997) Lipoprotein subfraction changes in normal pregnancy: threshold effect of plasma triglyceride on appearance of small, dense low density lipoprotein. J Clin Endocrinol Metab 82, 2483-2491.

50. Flock MR, Green MH \& Kris-Etherton PM (2011) Effects of adiposity on plasma lipid response to reductions in dietary saturated fatty acids and cholesterol. Adv Nutr $\mathbf{2}$, 261-274.

51. Moreno JA, Perez-Jimenez F, Marin C, et al. (2004) The effect of dietary fat on LDL size is influenced by apolipoprotein $\mathrm{E}$ genotype in healthy subjects. J Nutr 134, 2517-2522.

52. Campos H, D'Agostino M \& Ordovas JM (2001) Gene-diet interactions and plasma lipoproteins: role of apolipoprotein $\mathrm{E}$ and habitual saturated fat intake. Genet Epidemiol 20, $117-128$.

53. Ordovas JM (2001) Gene-diet interaction and plasma lipid response to dietary intervention. Curr Atheroscler Rep 3, 200-208.

54. Simopoulos AP, Leaf A \& Salem N Jr (1999) Essentiality of and recommended dietary intakes for omega- 6 and omega- 3 fatty acids. Ann Nutr Metab 43, 127-130.

55. Hibbeln JR, Nieminen LR, Blasbalg TL, et al. (2006) Healthy intakes of $n-3$ and $n-6$ fatty acids: estimations considering worldwide diversity. Am J Clin Nutr 83, Suppl. 6, 1483S-1493S.

56. Hodson L, Skeaff CM \& Fielding BA (2008) Fatty acid composition of adipose tissue and blood in humans and its use as a biomarker of dietary intake. Prog Lipid Res 47, 348-380.
57. Chowdhury R, Warnakula S, Kunutsor S, et al. (2014) Association of dietary, circulating, and supplement fatty acids with coronary risk: a systematic review and meta-analysis. Ann Intern Med 160, 398-406.

58. Wang DD, Li Y, Chiuve SE, et al. (2016) Association of specific dietary fats with total and cause-specific mortality. JAMA Intern Med 176, 1134-1145.

59. Estruch R, Ros E, Salas-Salvado J, et al. (2013) Primary prevention of cardiovascular disease with a Mediterranean diet. $N$ Engl J Med 368, 1279-1290.

60. Jakobsen MU, O'Reilly EJ, Heitmann BL, et al. (2009) Major types of dietary fat and risk of coronary heart disease: a pooled analysis of 11 cohort studies. Am J Clin Nutr 89, $1425-1432$

61. Jenab M, Slimani N, Bictash M, et al. (2009) Biomarkers in nutritional epidemiology: applications, needs and new horizons. Hum Genet 125, 507-525.

62. Nestel P, Clifton P \& Noakes M (1994) Effects of increasing dietary palmitoleic acid compared with palmitic and oleic acids on plasma lipids of hypercholesterolemic men. $J$ Lipid Res 35, 656-662.

63. Zhang C, Schulze MB, Solomon CG, et al. (2006) A prospective study of dietary patterns, meat intake and the risk of gestational diabetes mellitus. Diabetologia 49, 2604-2613.

64. Fung TT, Schulze M, Manson JE, et al. (2004) Dietary patterns, meat intake, and the risk of type 2 diabetes in women. Arch Intern Med 164, 2235-2240.

65. Micha R, Michas G \& Mozaffarian D (2012) Unprocessed red and processed meats and risk of coronary artery disease and type 2 diabetes an updated review of the evidence. Curr Atheroscler Rep 14, 515-524.

66. Wang X, Lin X, Ouyang YY, et al. (2016) Red and processed meat consumption and mortality: dose-response meta-analysis of prospective cohort studies. Public Health Nutr 19, 893-905. 